\title{
Traditional open surgery for advanced benign nasal tumours in an era of endoscopy: review of 38 cases.
}

\author{
Akeem O Lasisi* and Aderemi A Adeosun \\ Department of Otorhinolaryngology College of Medicine, University of Ibadan, P. O. Box 22040, University of \\ Ibadan, Ibadan, Nigeria. \\ *Author for Correspondence; E-mail: sakeemng@yahoo.com
}

\begin{abstract}
SUMMARY
During the past decade open nasal surgery has been overtaken by endoscopic resection in the management of benign sinonasal tumours in the advanced countries. However, due to limited availability of endoscopic surgical facilities and training in most parts of subSaharan Africa, open surgery still seems popular. This report determines the indications and outcome of open nasal surgery for benign tumours in a resource - poor tertiary centre in sub-Saharan Africa and compares with endoscopic resection. This is retrospective study of 38 open surgeries done by the authors for cases of benign sinonasal tumours. Seventeen (45\%) caldwel-luc procedures, 12 (31\%) external (lynch-howarth) fronto-ethmoidectomy and $9(24 \%)$ lateral rhinotomy were carried out. The indications were simple nasal polyps $17(45 \%)$, mucocoele $10(26 \%)$, inverted paplloma $8(21 \%)$, invasive fungal sinusitis $2(5 \%)$ and ossifying fibroma 1(2\%). Postoperatively, recurrence was $1 / 17(6 \%)$ caldwel-Luc surgery and $1 / 9(11 \%)$ lateral rhinotomy for inverted papilloma. Facial scar was seen in $21 / 38(55 \%)$ but the cosmetic effect of the scars was acceptable to our patients. Postoperative stay was between 5 to 17 days. We conclude that open nasal surgery met the therapeutic expectations of our patients. The recurrence rate compared with that of endoscopic resection reported in the literature and cosmetic effect of facial scar was acceptable to the patients, however, postoperative stay in the hospital is longer. It is our hope that this will serve as a baseline to compare the result of endoscopic nasal surgery in future when it becomes a widespread procedure in the developing countries.
\end{abstract}

$$
\text { [Afr J Health Sci. 2007; 14: 44-48] }
$$

\section{Introduction}

The external approach has been the traditional route for management of chronic sinonasal masses until the late 1980s when technological advances brought endoscopy and has since been increasing in popularity. This has raised a comparison of the two modalities, presence of residual or recurrent disease and facial scar being the clinical indices most commonly considered. The success rate of endoscopic resection reported varied widely between 21 to $98 \%[1,2,3]$ some authors reported comparable result between endoscopic and open surgery [3,4]. The extent of disease and surgical expertise were confounding factors. In the subsaharan Africa, delayed presentation leading to advanced disease stage is routine. This and the limited availability of endoscopy further strengthen the need for proficiency in open nasal procedures. We report the spectrum of the indications and the outcome of our open surgical excision for benign sino-nasal tumours, compare with reports of endoscopic resection inorder to evaluate the benefits derived by our patients. It will also serve as a reference for the outcome assessment of our endoscopic sinus surgery in future.

\section{Method}

This is a retrospective analysis of 38 surgical procedures done on 32 patients with benign sinonasal masses within the last 4 years (2000-2004). Data from the clinical records of the patients were retrieved for surgical indications, operative findings histopathology and outcome. All the cases were done by the two authors under general anesthesia administered via orotracheal intubation. The classical approach was used in all the cases. The lateral rhinotomy incision is a skin crease longitudinal incision from a point just below the medial palpebral ligament through the nasomaxilary grove. It curved round the alar to enter the nose (Figure 1a). It exposes the nasal bone, cavity, anterior ethmoid and anteromedial wall of the maxillary antrum. Osteotomy depends on the extent of disease but may include removal of the anteromedial wall of the 
maxillary antrum and anterior ethmoid, frontonasal process, lacrimal fossa and lamina papyracea. After Fig 1: Incision wound closure in immediate postoperative period

1 (a)

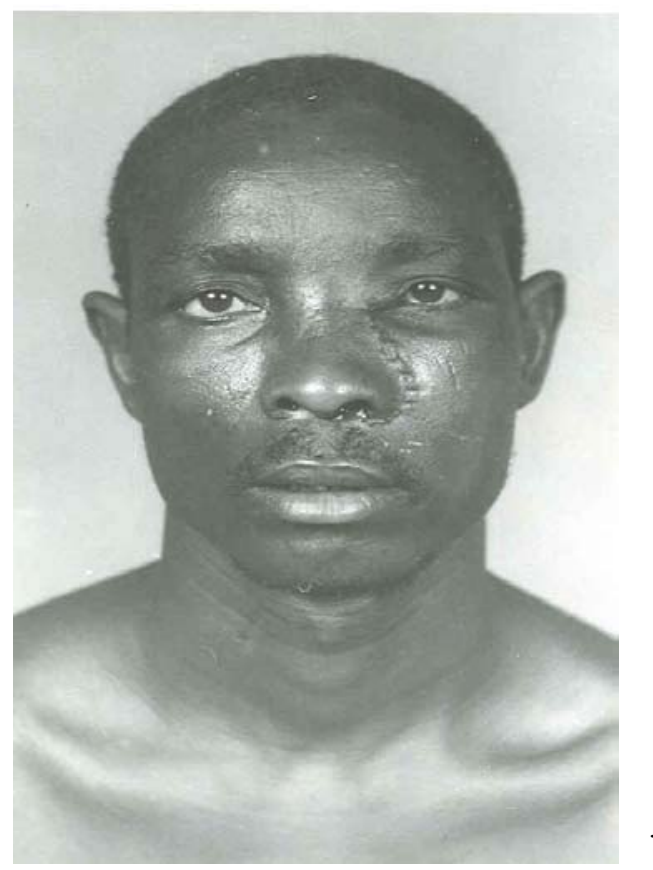

excision of the lesion, the mucosa, periosteum and skin were closed.

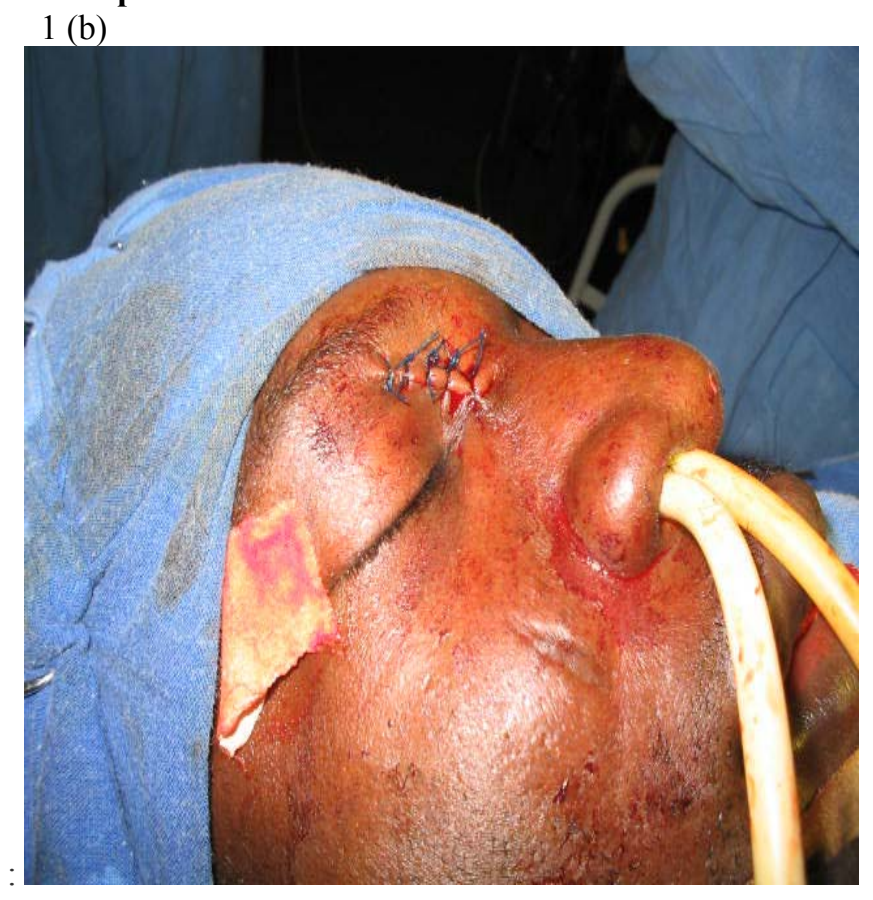

1(a): Left lateral rhinotomy incision;

1(b): Left frontoethmoidectomy incision wound with folley's catheter frontonasal stent insitu

The Lynch-Howarth incision was placed in the superomedial angle of the orbit, avoiding the trochlear nerve and preserving the lateral support of the frontal recess (Fig 1b). The periosteum was removed and access gained into the frontal sinus. This allows maximum but safest marsupialization of the frontal sinus mucosa. A frontonasal stent was put in place for 14 days (Fig 1b). The caldwel-luc antrostomy surgery involved a sublabial incision and osteotomy was done to enter the maxillary antrum. An antral catheter was left in situ for 24-48hours. All the patients had a follow-up period between 17 to 24 months.

\section{Results}

A total number of 38 open nasal procedures were done on 32 patients during the period of study. There were 17 males and 15 females (1.2:1) and the age ranged between 16 to 72 years with a mean of 41 years. The common clinical features were persistent nasal blockage (28), rhinorrhoea (26) and headache and trigemial pain (8). Proptosis (14), facial deformity (9) and epistaxis (7) were seen in advanced cases (Fig 2a, b). All the patients had plain radiograph while only 11 had computerized axial tomography scanning (CT Scan). This revealed localized opacity in the maxillary antrum in (7), frontal sinus (4), multiple sinus involvement in (21), destruction of bony walls of the sinuses (16) and destruction of the sphenoid and cribriform plate (6) and lamina papyracea (5) (Fig 3). Using the Mackay and Lund $^{5}$ staging of nasal polyps, all the polyps were stage 3 .

\section{Caldwel-luc antrostomy surgery}

This was done in 17 patients. The indications were 13 polyps, 2 invasive fungal sinusitis and 1 mucocoele. Operative findings were dumb-bell extension into the nasal cavity in 3, with destruction of the medial wall of the antrum in 4 and involvement of the floor of the orbit in 2. Encysted thick jelly-like secretion appearing like mucocoele was found in 3. The patients were discharged between 5 and 10 days after surgery. Postoperative complications seen were pain and facial swelling that subsided within 5 days. Residual disease was found in 1 patient. Paraesthesia of the upper teeth was found in 9, it resolved in 3 patients between 8- 14 months. Histopathology revealed simple polyp in 6 , pyogenic polyp in 7 , invasive fungal sinusitis and allergy in 2 and mucocoele in 1 . 
Fig 2: Clinical photograph of the anteroposterior view of the face 2(a)

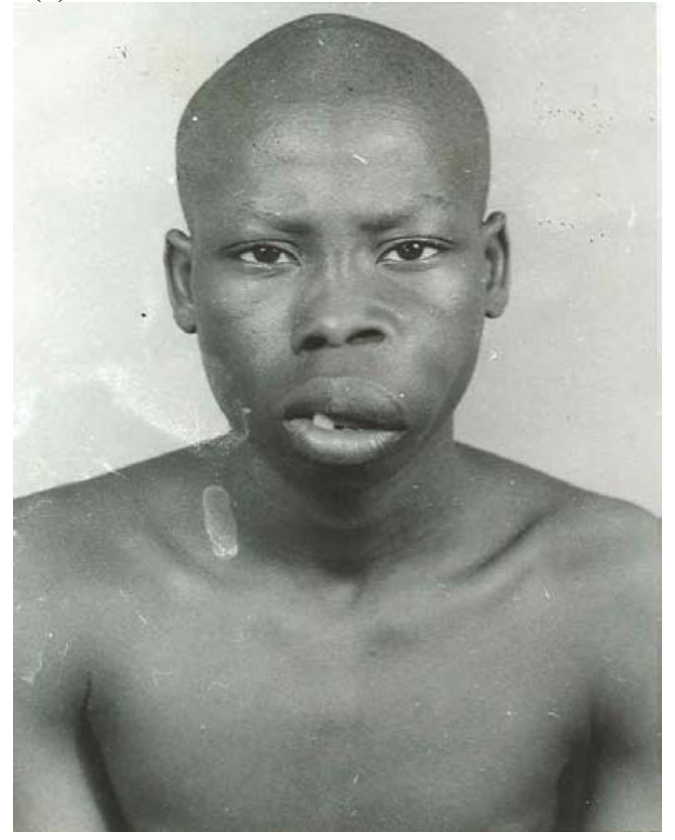

2(b)

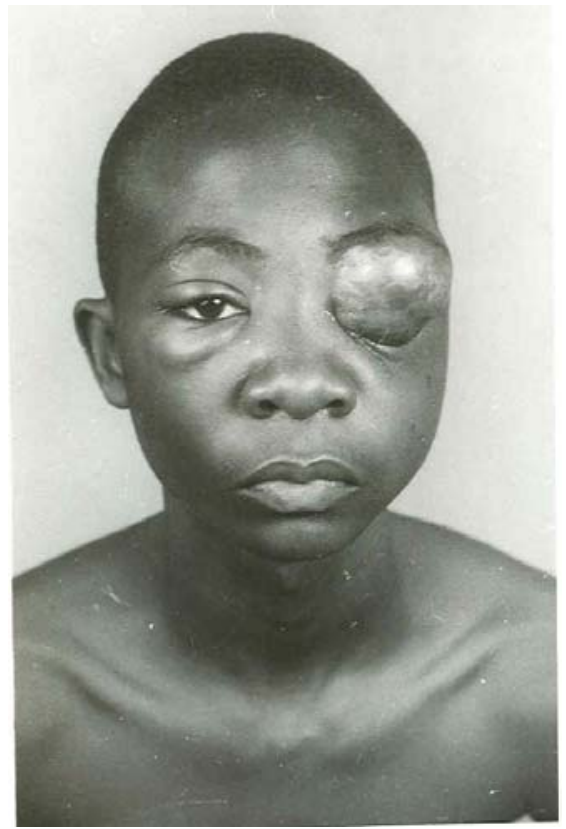

2(a): Shows circumscribed left hemifacial swelling. It is firm to hard in consistency. The patient had left medial maxillectomy through a left lateral rhinotomy incision.

2(b): Shows ptosis and proptosis of the left eye resulting from orbital cellulites complicating acute on chronic rhinosinusitis.

\section{External (Lynch-Howarth) Fronto- Ethmoidectomy}

Twelve cases were done. The indications were frontal mucocoele in 9 and pyocoele in 1. The operative findings included encysted mucus collection in the frontal sinus at times mixed with pus, ethmoidal polyps in 8 , destruction of the lamina papyracea was seen in 4 , defect in the posterior wall of the frontal sinus with communication with the anterior cranial fossa in 2. Facial pain and swelling resolved within 3 days after surgery, facial scar in 13 , became unnoticeable after 18 months in 4 and 1 had hypertrophy of the scar.

\section{Lateral rhinotomy}

Nine cases were done. The indications were inverted papiloma 8 and 1 ossifying fibroma. The findings at surgery were multilobulated grape-like nasal masses, it was bilateral in 1 patient. The ethmoids and maxillary antrum were involved in all, nasal bone and bony septum in 5, sphenoidal sinus in 3 and frontal sinus in 2. Recurrence of papilloma was seen in 1 patient and facial scar in all.

\section{Discussion}

Benign nasal polyps accounted for the commonest indication $23 / 38$ for open nasal surgery. This is in contrast to the review of the literature that reported inverted papilloma and mucocoele $\left[\begin{array}{ll}2,3 & 6,7\end{array}\right]$. The polyps seen were advanced, they were Grade 3 Mackay and Lunds [5] and also involve multiple regions with associated bony destruction; mucocoele and pyocoele formation. The goal in management of benign nasal disease is complete excision with preservation of the mucosa. Comparison between external approach and endoscopy is difficult. The factors influencing result include extent of disease, adequate visualization, predisposing factors, radiologic evidence of preoperative bone destruction, previous treatment, individual patient factor, surgeon preference and experience [8,9,10]. In our experience, we were able to achieve reasonably complete excision in most cases. 
Fig 3: Computerized axial tomography scan of the paranasal sinuses

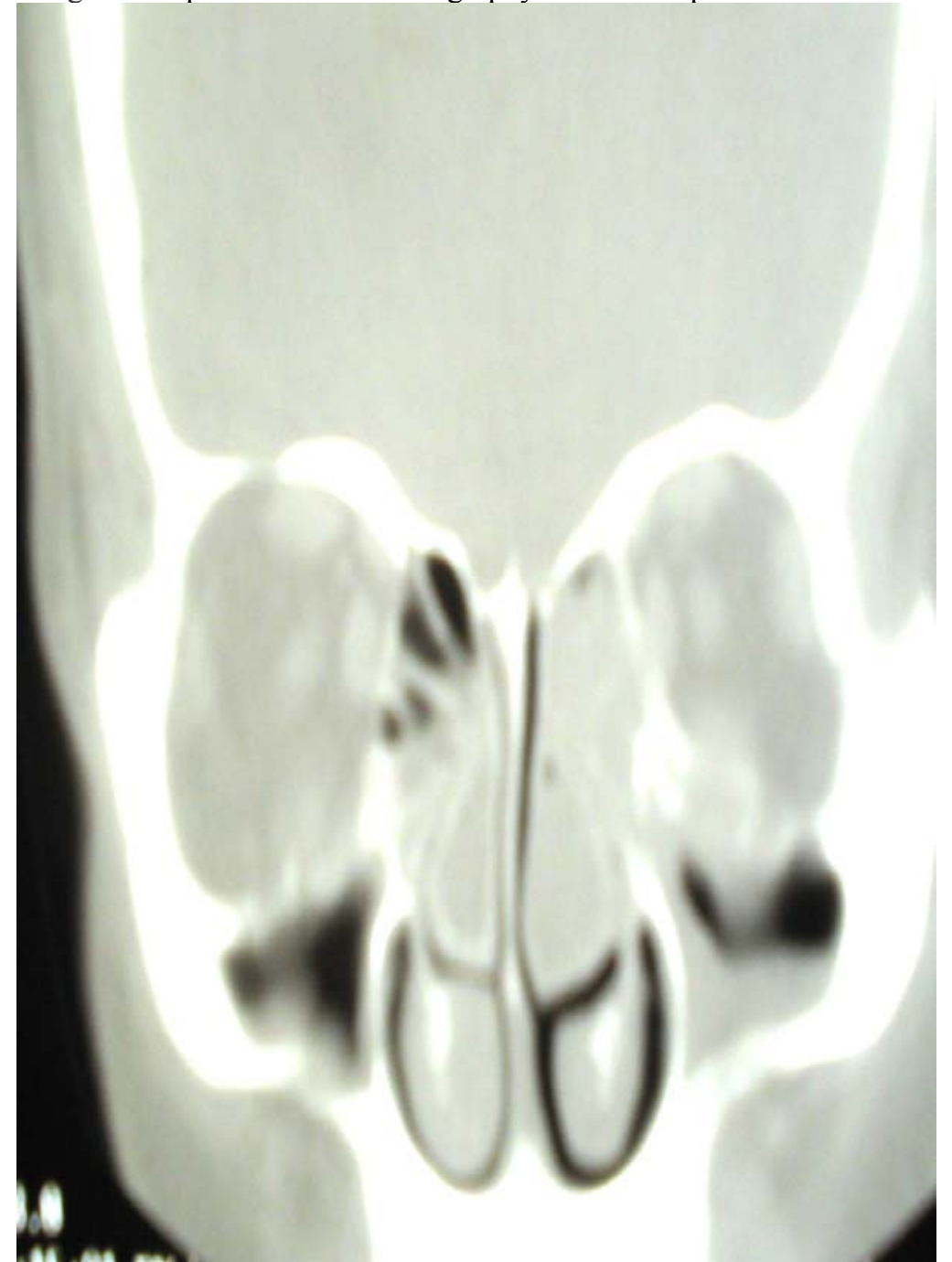

Fig 3: Shows soft tissue shadow density involving both nasal cavity, ethmoids, maxillary and sphenoidal sinuses, there is destruction of bones of the sphenoid, ethmoids and posterior half of the cribriform bone.

We had recurrence1/17 (6\%) caldwel-Luc antrostomy surgery for maxillary polyp and $1 / 9(11 \%)$ lateral rhinotomy for papilloma. The recurrence was associated with the presence of other pathologic processes such as allergy in the patient which in our opinion added to the pathologic process of mucocoele formation $[9,10,11]$ Mucocoele is an epithelial -lined mucus - containing sac completely filling a paranasal sinus and capable of expansion by dynamic process of bone resorption and new bone formation. The frontal sinus are most frequent followed by sphenoid and maxillary. Maxillary antral involvement and positive bacterial culture have been reported as predictive criteria of recurrence [11]. Our recurrence is similar to that of other workers $[1,2,3]$ who reported recurrence rate after lateral rhinotomy between $0-29 \%$, an average of $14 \%$ and endoscopy; range of $0-27 \%$, average of $18 \%$. Lund [1] reported
37 cases of inverted papilloma between $1992-98$, recurrence was $1 / 13(8 \%)$ for endoscopy and $5 / 24$ (21\%) for combined approach, thus concluded that recurrence was related to extent of disease. Watz and Wigand [12] also reported that recurrence is related to stage of disease, in their report recurrence in $\mathrm{T} 1$ and 2 was $1 / 20(5 \%), \mathrm{T} 3$ and 4 was $5 / 15(30 \%)$ following external nasal approach after 46 month follow-up. Sukenic and Casian [13] reported recurrence of $21 \%$ in 19 patients, Tuffanol et al [14] reported a $6 \%$ and $25 \%$ recurrence after endoscopy for primary and secondary disease. We had recurrence following the Lynch Howarth (frontoethmoidectomy) procedure for resection of mucocoele. In a report of 48 mucocoeles, 20 treated by an entirely endoscopic approach and 28 by combined external and endoscopy, Lund [2] reported no recurrence with endoscopy and 3 
recurrences with the combined group, Ikeda et al [6] reported no recurrence in a series of 97 patients with mucocoele treated with endoscopy and external approach after 36-84 month follow-up period. Kennedy et al [9] reported endoscopic marsupialization in 18 cases without recurrence. Busaba and others [7] have reported similar favorable result with endoscopy alone. In a cohort of 118 treated with Lynch incision between 1965-1990, 98\% \% were ethmoidal, $1 \%$ sphenoidal and maxillary, between 1991-1996, 48 cases were done with endoscopy and combined procedure. Rubin [15] reported webbing of the scar in $6 \%$ in a review of 60 patients. The main drawback of external approach seems to be the facial scar and prolonged postoperative stay in the hospital. Facial scar was seen in $21 / 38$, however, the scar became unnoticeable in about $50 \%$ within 15 months and, cosmetic effect of the scar seem acceptable to our patients. This is similar to the report of Lund et al [1]. Extent of disease is determined by the evaluation of the clinical, radiologic and general condition of the patient. In this report, CT Scan was available in only about $30 \%$ of cases due to financial cost of the investigation. The implication of this is limitation to the accuracy of clinical judgement and decision. This is particularly relevant in the assessment of base of the skull. Authors with bias for endoscopy have reported involvement of the frontal sinus, lateral maxillary wall, nasolacrimal duct and skullbase as reason for incomplete excision in external approach.

\section{Conclusion}

It was noticed that the patients in our series presented with advanced disease. Despite this, the outcome, in terms of recurrence and cosmesis appeared comparable to reports on endoscopic resection. This may be related to the techniques of the operators. Surgical dexterity has also been reported among the determinants of outcome although it has always been difficult to assess and standardize. It will ensure a complete excision of disease with minimal injury to sensitive structures in the base of the skull. However, it is our hope to compare this result with endoscopy in future when it becomes established in our subregion..

\section{References}

1. Lund VJ. Optimum management of inverted papilloma. Journal of Laryngology and Otology. 2000; 114: 194-197.

2. Lund VJ. Endoscopic management of paranasal sinus mucocoele Journal of Laryngology and Otology. 1998; 112:36-40.
3. Lessa MM, Marcondes R.A, Goto EY, Romano F, Voegels RL \& Butugan O. Endoscopic endonasal surgery: Experience in 706 cases. Revista Brasileira de Otorrinolaringologia (English translation) 2001; 67:513-517.

4. Casiano R R. Transnasal endoscopic surgery for benign neoplasms of the nose and sinuses. Current Opinion in otorhinolaryngology \& Head and Neck Surgery. 2001; 9:37-41.

5. Mackay IS and Lund VJ: Imaging and staging. In nasal polyposis: An Inflammatory Disease and Its Treatment. Edited by Mygind N, Lildholdt T. Copenhagen: Munksgaard; 1997: 137 - 144.

6. Ikeda K, Takahashi C, Oshima T,Suzuki H, Satake M, Hidaka H, Takasaka T. Endonasal endoscopic marsupialization of paranasal sinus mucocoele. American Journal of Rhinology. 2000; 14: 107-111.

7. Busaba NY, Salman SD: Maxillary sinus mucocoeles: Clinical presentation and long term result of endoscopic surgical treatment. Laryngoscope. 1999; 109: 1446-1449.

8. Assanasen $\mathrm{P} \&$ Naclerio RM: Medical and surgical management of nasal polyps. Current Opinions in Otorhinolaryngology \& Head and Neck Surgery. 2001; 9: 27-36.

9. Kennedy DW, Josephson JS, Zinreich SJ, Mattox D E, Goldsmith MM. Endoscopic sinus surgery for mucoceles: A viable alternative. Laryngoscope. 1989; 99: 885-895.

10. Moriyama H, Nakajima T, Honda Y. Studies on mucocoeles of ethmoid and sphenoid sinuses: Analysis of 47 cases. Journal of Laryngology and Otology. 1992; 106: 23- 27.

11. Bailey BJ, Pillsburg HC, Driscall BE: Head and Neck surgery - Otolaryngology, Second Edition, Chapters 35-37, 1998.

12. Waitz G \& Wigand ME: Result of endoscopic sinus surgery for the treatment of inverted papillomas. Laryngoscope. 1992; 102: 917-922.

13. Sukenik MA, Casiano R. Endoscopic medial maxillectomy for inverted papilloma of the paranasal sinuses: value of intraoperative endoscopic examination. Laryngoscope. 2000; 110: $39-42$.

14. Tufano RP, Thaler ER, Lanza DC, Goldberg AN, Kennedy DW. Endoscopic management of sinonasal inverted papilloma. American Journal of Rhinology.. 1999; 13:423-426.

15. Rubin JS, Lund VJ, Salmon B. Frontoethmoidectomy in the treatment of mucocoeles. A neglected operation. Archives of Otolaryngology - Head and Neck Surgery. 1986; 112:434-436. 